\title{
KONDO-LATTICE METALS AND SEMICONDUCTORS WITH COMPENSATED MAGNETIC MOMENTS: A BRIEF OVERVIEW
}

\author{
J. SPALEK ${ }^{a}$ AND R. DoRADZIŃSKI ${ }^{b}$ \\ ${ }^{a}$ Marian Smoluchowski Institute of Physics, Jagiellonian University \\ Reymonta 4, 30-059 Kraków, Poland \\ ${ }^{b}$ Institute of Theoretical Physics, Warsaw University, Hoża 69, 00-681 Warszawa, Poland
}

We provide a brief overview of recent theoretical results concerning the metallic and insulating states with very heavy quasiparticle masses and almost compensated magnetic moments, primarily in the antiferromagnetic state. The temperature dependence of the Kondo-insulator band gap is also discussed.

PACS numbers: 71.27.+a, 71.10.Fd, 75.30.Mb

\section{Introduction}

The heavy-fermion systems have been studied intensively during the two last decades. The interest started with the discovery [1] that the metallic system $\mathrm{CeCu}_{2} \mathrm{Si}_{2}$ with the heavy quasiparticles of mass $m^{*} \sim 10^{3} m_{0}$, where $m_{0}$ is the free-electron mass, are superconductors below the temperature $T_{\mathrm{S}} \approx 1 \mathrm{~K}$. The interest in those systems was also stimulated by two further findings. First [2], some of those systems such as $\mathrm{Ce}_{3} \mathrm{Bi}_{4} \mathrm{Pt}_{3}$ or $\mathrm{YbB}_{12}$ are insulating at temperature $T=0 \mathrm{~K}$, but become heavy-fermion metals with increasing temperature. They are called the Kondo insulators. Second [3], several metallic systems exhibit strong deviations from the Fermi-liquid behavior at $T<1 \mathrm{~K}$. Namely, one encounters the behavior of the linear-specific-heat coefficient $\gamma(T) \equiv C(T) / T \sim \ln \left(T_{0} / T\right)$, the dependence for the magnetic susceptibility $\chi(T) \sim \chi_{0}(1-\alpha \sqrt{T})$, and the resistivity behavior $\rho \simeq \rho_{0}+A^{\prime} T$, where $T_{0}, \chi_{0}, \alpha, \rho_{0}$, and $A^{\prime}$ are constants. These temperature dependences are commonly termed a non-Fermi liquid behavior (strictly, they should be called a non-Landau behavior of fermionic liquids).

One should recognize at the outset a fundamental significance of those results. First, the electrons, almost as heavy as the nucleons, should be regarded as being very close to the Mott localization point (at which the electrons in the localized or atomic states acquire infinite effective mass for translational motion throughout the system). The fact that they are almost localized (i.e. have large density of states at the Fermi level) means that they have well defined magnetic 
moments. Instead, the heavy quasiparticles deriving from $5 f$-electrons in the systems such as $\mathrm{UPt}_{3}$ or $\mathrm{URu}_{2} \mathrm{Si}_{2}$ have a very small staggered moment of the order of few parts in hundred of the Bohr magneton, per $U$ atom. At the same time, they superconduct at the temperature $T_{\mathrm{S}} \sim T_{\mathrm{N}} / 10$, where $T_{\mathrm{N}}$ is the Neel temperature ( $5 \mathrm{~K}$ and $17 \mathrm{~K}$, respectively). Parenthetically, one can also expect that the superconductivity must be of spin-singlet type to make it in agreement with antiferromagnetic nature of the spin ordering. Furthermore, the non-Fermi liquid behavior has been detected in a clear form in the system such as $\mathrm{UCu}_{5-x} \mathrm{Pd}_{x}$ [3], where the atomic (substitutional) disorder and the proximity of the transition to the antiferromagnetic phase are regarded to play an important role at the same time. Theoretically, the non-Fermi liquids are regarded as the systems close to the quantum (magnetic) critical point. By this we understand that the microscopic parameters of the system are such that their small change triggers the magnetic ordering at $T=0$ (in the ground state, when thermodynamic fluctuations are absent).

Our task [4] was to look at the metallic and insulating heavy fermion systems from the magnetically ordered side and seek for the inherent mechanism of the magnetic moment reduction, if not for its total compensation. In those systems one has both conduction $(c)$ and atomic $(f)$ bare states. The question then is if the Kondo-like compensation (albeit, in the periodic lattice situation) is sufficient to explain the existence of very low magnetic moments. It turns out that this mechanism in combination with the Fermi-liquid nature of $f$-electrons can satisfactorily explain that effect. We provide also the temperature dependence of the Kondo insulating gap. The Kondo insulators (semiconductors at $T>0$ ) should be regarded as a new class of semiconductors, for which the gap gradually reduces to zero without any magnetic transition accompanying it. Therefore, it should be distinguished from the Mott or Slater insulators or antiferromagnetic semiconductors. In brief, the Kondo insulators represent a new class of semiconductors. In the next two sections we overview our results [4] from a physical point of view.

\section{Heavy fermion metals with compensated moments}

The discussion of the heavy fermion system is based on the Anderson-lattice Hamiltonian or its derivatives. It has the following form:

$$
\begin{aligned}
H= & \sum_{i j \sigma}^{\prime} t_{i j} c_{i \sigma}^{\dagger} c_{j \sigma}+\varepsilon_{f} \sum_{i \sigma} a_{i \sigma}^{\dagger} a_{i \sigma}-\frac{1}{2} g_{c} h \cdot\left(\sum_{i \sigma \sigma^{\prime}} c_{i \sigma}^{\dagger}(\tau)_{\sigma \sigma^{\prime}} c_{i \sigma^{\prime}}\right) \\
& -\frac{1}{2} g_{f} h \cdot\left(\sum_{i \sigma \sigma^{\prime}} a_{i \sigma}^{\dagger}(\tau)_{\sigma \sigma^{\prime}} a_{i \sigma^{\prime}}\right)+V \sum_{i \sigma}\left(c_{i \sigma}^{\dagger} a_{i \sigma}+a_{i \sigma}^{\dagger} c_{i \sigma}\right) \\
& +U \sum_{i} a_{i \uparrow}^{\dagger} a_{i \uparrow} a_{i \downarrow}^{\dagger} a_{i \downarrow},
\end{aligned}
$$

where $c_{i \sigma}^{\dagger}$ and $a_{i \sigma}^{\dagger}$ are the creation operators of the conduction (c) and localized $(f)$ electron states, respectively, the indices $i$ and $j$ label the lattice sites and $\sigma$ - their spin quantum number, $\sigma= \pm 1$, whereas $h \equiv \mu_{\mathrm{B}} \boldsymbol{H}_{a}$ denotes the applied magnetic field rescaled by the Bohr magneton $\mu_{\mathrm{B}}$. 
The third term in (1) describes the band motion of conduction electrons, here represented as a hopping from site $j$ to $i$, with the hopping matrix element $t_{i j}$. Applying the space Fourier transform to the creation $\left(c_{i \sigma}^{\dagger}\right)$ and annihilation $\left(a_{i \sigma}\right)$ operators, this term takes the usual band form $\sum_{k_{\sigma}} \varepsilon_{k} a_{k_{\sigma}}^{\dagger} a_{k_{\sigma}}$, where $k$ is the wave vector of a single electron and $\varepsilon_{k}=N^{-1} \sum_{i j} t_{i j} \exp \left[i k \cdot\left(\boldsymbol{R}_{i}-\boldsymbol{R}_{j}\right)\right]$ is its band energy. The second term describes the atomic energy of $f$-electrons, each placed on the $f$-level positioned at $\varepsilon_{F}$. The third and the fourth terms describe the Zeeman energy of the two subsystems ( $c$ and $f$, respectively). The fifth term represents the hybridization (i.e. the intraatomic nonorthogonality of the Wannier orbitals) of the $c$ - and $f$-electrons. Finally, the last term describes the intraatomic Coulomb energy if two $f$-electrons meet on the same orbital (we do not take into account the orbital degeneracy for the sake of simplicity). The situation is schematically represented in Fig. 1, where the division into low-energy $\left(\sim V /\left(\varepsilon_{f}-\mu\right)\right)$ and high-energy $\left(\sim V /\left(\varepsilon_{f}+U\right)\right)$ processes is also made.

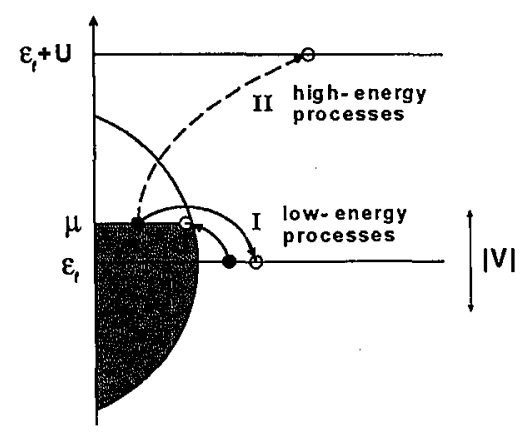

Fig. 1. Division of the hybridization ( $f-c$ mixing) into the high-energy processes $\left(\sim V /\left(\varepsilon_{f}+U-\mu\right)\right)$ and the low-energy mixing $\left(\sim V /\left(\varepsilon_{f}-\mu\right)\right)$. These processes combined together lead in the second order to the Kondo coupling.

The Hamiltonian (1) describes the simplest situation with the two subsets of electrons, which hybridize with each other (exchange electrons) and in the situation when one of them composes a system of atomic and strongly interacting electrons, while the other represents noninteracting charge carriers. Such a model Hamiltonian possesses two trivial limits, namely the limit $V \equiv 0$, when the $f$-electrons are completely decoupled from the carriers and form a subsystem of localized spins, and the limit $U=0$, when the noninteracting $f$ - and $c$-electrons form two (bonding and antibonding) bands.

Further analysis of the Hamiltonian is performed using the auxiliary (slave) boson representation and by making the saddle-point approximation. The details of the technique have been discussed before $[5,4]$. Therefore, we limit our discussion here to presenting the main physical results.

The heavy fermion systems represent the strongly correlated electrons, for which the Coulomb interaction $U$ is regarded as the highest energy scale of the system $(U \gg|V|$ or $W$, where $W=2 z|t|$ is the width of the bare-band states). In this limit the $f-c$ quantum mechanical mixing of the states is reduced due to the cir- 
cumstance that the doubly occupied $f$-level configurations are strongly suppressed. This means that the hybridization matrix element is renormalized and spin dependent, $V \rightarrow \tilde{V}_{\sigma}=V q_{\sigma}$, where $q_{\sigma}$ is to be found self-consistently. Probably it is even more difficult to grasp intuitively the fact that the bare $f$-level position is strongly renormalized by the interaction, $\varepsilon_{f} \rightarrow \varepsilon_{f}+\beta_{0}$, so that $\tilde{\varepsilon}_{f}-\mu \equiv k_{\mathrm{B}} T_{\mathrm{K}} \ll\left|\mu-\varepsilon_{f}\right|$, where $k_{\mathrm{B}} T_{\mathrm{K}}$ is a new energy scale appearing in the system. The quantity $T_{\mathrm{K}}$ is called the effective Kondo temperature (strictly speaking, it should be called the effective hybridization, in temperature units). Finally, a strong, nonlinear molecular field (with the $z$-component denoted by $\beta_{3}$ ) appears in the system, so that in the uniform magnetically polarized state the quasiparticle energies are

$$
\begin{aligned}
E_{\boldsymbol{k}_{\sigma}}^{\mathbf{s}} & =\frac{1}{2}\left[\varepsilon_{k}+\varepsilon_{f}+\beta_{0}+\sigma \beta_{3}-\frac{1}{2} \sigma\left(g_{c}+g_{f}\right) h_{3}\right. \\
& +s \sqrt{\left.\left[\varepsilon_{k}-\varepsilon_{f}-\beta_{0}-\sigma \beta_{3}-\frac{1}{2} \sigma\left(g_{c}-g_{f}\right) h_{3}\right]^{2}+4\left|\tilde{V}_{\sigma}\right|^{2}\right]} .
\end{aligned}
$$

The symbol $s= \pm 1$ labels the hybridized bonding and antibonding states of fermionic quasiparticles, and $h_{3}$ is the $z$-component of the applied field. We have two bands, so the $f$-electrons acquire itineracy induced by the hybridization. Obviously, the expression (2) represents energies which are still to be adjusted by minimization of the free-energy $(T>0)$ or ground-state-energy $(T=0)$ functionals. Such a procedure involves a minimization over number of parameters and usually is performed numerically (see below). However, the paramagnetic state can be analyzed analytically and this example will be discussed before going to the magnetic states.

\subsection{Case A: paramagnetic metallic state}

The Kondo-lattice state representing the heavy-fermion state will be understood as the limit

$\frac{|V|}{W}, \quad$ and $\quad \frac{V}{\varepsilon_{f}-\mu} \ll 1$.

Both inequalities amount to the physical limit in which the starting atomic states hybridize weakly with the carrier states and that the atomic level is placed relatively deeply below the Fermi level. In other words, the $f$-electrons are almost localized even in this environment of extended states with which they hybridize. Under these assumptions the quasiparticle states with energies (2) in the non-polarized state, i.e. with

$$
E_{k \sigma}^{\mathbf{s}} \equiv E_{k}^{\mathbf{s}}=\frac{1}{2}\left(\varepsilon_{k}+\tilde{\varepsilon}_{f}\right)+\frac{s}{2} \sqrt{\left(\varepsilon_{k}-\tilde{\varepsilon}_{f}\right)^{2}+\tilde{V}^{2}},
$$

have the density of states

$$
\rho^{\mathrm{s}}(E)=\rho_{0}\left[1+\frac{\tilde{V}^{2}}{\left(E-\tilde{\varepsilon}_{f}\right)^{2}}\right]
$$


where $\rho_{0}$ is the density of states in the bare band. The gap between the hybridized bands is equal to

$$
\Delta_{\mathrm{G}}=2 k_{\mathrm{B}} T_{\mathrm{K}} \equiv 2\left(\tilde{\varepsilon}_{f}-\mu\right)=\varepsilon_{f}+\beta-\mu \simeq \frac{\tilde{V}^{2}}{W} .
$$

Also, the density of states (4) at the Fermi level is

$$
\rho(E=\mu) \simeq \frac{\tilde{V}^{2}}{W} \frac{1}{\left(\tilde{\varepsilon}_{f}-\mu\right)^{2}}=\frac{1}{2 k_{\mathrm{B}} T_{\mathrm{K}}},
$$

where $\rho_{0}=1 / W$, i.e. is assumed to be constant in the range $-W / 2 \leq \varepsilon \leq W / 2$. Therefore, the effective Kondo temperature can be measured directly from the linear (molar) specific heat, since $C_{V} \equiv \gamma T$, and $\gamma=\frac{2}{3} \pi^{2} k_{\mathrm{B}}^{2} \rho(\mu) N_{\mathrm{Av}}=\frac{2 \pi^{2}}{3} R \frac{1}{T_{\mathrm{K}}}$. For example [6], for $\mathrm{CeRu}_{2} \mathrm{Si}_{2} \gamma=355 \mathrm{~mJ} /\left(\mathrm{mol} \mathrm{K}^{2}\right)$ and we obtain that $T_{\mathrm{K}} \approx 80 \mathrm{~K}$. The most important at this stage is the circumstance that $T_{\mathrm{K}}$ can be expressed by the model parameters, namely that

$$
k_{\mathrm{B}} T_{\mathrm{K}}=\frac{W}{2} \exp \left(-\frac{\left|\varepsilon_{f}\right|}{2 \rho_{0} V^{2}} \frac{U-\left|\varepsilon_{f}\right|}{U}\right) .
$$

Note that the parameters $V \approx-0.1 \mathrm{eV}$ and $\varepsilon_{f} \approx-(1 \div 2) \mathrm{eV}$ are regarded negative, since we selected the middle point of the band energy as the zero of energy. From formula (8) we see that $k_{\mathrm{B}} T_{\mathrm{K}}$ is indeed a new scale of energy if the inequalities (3) are satisfied. We obviously have that $U-\left|\varepsilon_{f}\right|>0$, since for known systems $U \approx 5-6 \mathrm{eV}$. Then, taking $\varepsilon_{f}=-2 \mathrm{eV}, W=2 \mathrm{eV},|V|=0.3 \mathrm{eV}, U=6 \mathrm{eV}$, we obtain numerically that $T_{\mathrm{K}} \approx 3.5 \mathrm{~K}$. As a result, $\rho(\mu) \approx 2000$ states $/ \mathrm{eV}$ per site! Taking the value of the antiferromagnetic Kondo coupling $J$ from the Schrieffer-Wolff expansion [7], i.e.

$$
J=\frac{2 V^{2}}{\left|\varepsilon_{f}\right|\left(U-\left|\varepsilon_{f}\right|\right)}
$$

we can write down expression (8) in the form

$$
k_{\mathrm{B}} T_{\mathrm{K}}=\frac{W}{2} \exp \left(-\frac{1}{\rho_{0} J}\right) \text {. }
$$

This formula is close to that for the impurity Kondo effect [8]. However, in our present situation the meaning of $T_{\mathrm{K}}$ is quite different. In the impurity case the antiferromagnetic coupling $\left(J S_{f} \cdot S_{c}\right)$ between the carriers $(c)$ and atomic $(f)$ states arises from a virtual hopping from the $f$-level onto the Fermi surface and back or from the Fermi surface onto singly occupied $f$-level and back. These transitions are at least of the second order $\left(\sim \frac{V^{2}}{U}\right.$ and $\left.\sim \frac{V^{2}}{\varepsilon_{f}-\mu}\right)$ and are possible only if the virtually excited conduction electron occupying the same state has the opposing spin to the localized $(f)$ electron residing already there. In the lattice case the itineracy of $f$-electrons involves three-stage process: a transition from the $f$-level to the conduction band, followed by a propagation in the conduction band, and a subsequent deexcitation from the conduction band onto one of the $f$-levels on a neighboring site. One should notice that if the conduction-band state is envisaged as a plane wave, then the probability of finding the electron is the same on each lattice site and equal to $n_{f}$, whereas the probability of hopping is $n_{f}\left(1-n_{f}\right)$ (as in 
the large- $U$ limit the double occupancies are suppressed). As a result, the effective hopping integral for quasiparticle $f$-states is

$$
t_{f f} \sim \frac{V^{2}}{\varepsilon_{f}-\mu} n_{f}\left(1-n_{f}\right)
$$

with

$$
1-n_{f} \approx\left(\frac{U-\left|\varepsilon_{f}\right|}{U}\right)^{2} \frac{k_{\mathrm{B}} T_{\mathrm{K}}}{2 \rho_{0} V^{2}} \sim \frac{W}{V^{2}} k_{\mathrm{B}} T_{\mathrm{K}}
$$

so that for the parameters specified above $t_{f f} \sim k_{\mathrm{B}} T_{\mathrm{K}}$. Therefore, indeed the energy scale $T_{\mathrm{K}}$ expresses the effective band width of heavy quasiparticles of the $f$-type. Note that if the number of electrons per atom is $n \equiv n_{f}+n_{c}<2$, then the Fermi level lies in the lower hybridized band. Then, as we said $\rho(\mu)=1 /\left(2 k_{\mathrm{B}} T_{\mathrm{K}}\right)$, so that the sum rule concerning the number $\Delta N_{f}$ of quasiparticles in the $f$-states (per site) is $\Delta N_{f} \sim \rho(\mu) k_{\mathrm{B}} T_{\mathrm{K}} \sim 1$, i.e. almost all $f$-states are located near the Fermi level. This result should not be regarded as surprising, since the quasiparticle $f$-level is moved to the Fermi level $\left(\varepsilon_{f} \rightarrow \tilde{\varepsilon}_{f}=\varepsilon_{f}+\beta_{0}\right)$. Also, there is almost one $f$-electron per site, since $1-n_{f} \ll 1$ (see Eq. (12)).

Summarizing, the huge density of states arises from quasiparticle states deriving from the $f$-states. In other words, such large value for the density of states derives from the large effective masses, as close to the Fermi level $\mu$ and the Fermi wave vector $k_{\mathrm{F}}$ one can linearize the dispersion relation in the following way:

$$
E_{k}^{(-)}-\mu \simeq-k_{\mathrm{B}} T_{\mathrm{K}}-\left.\frac{\partial E_{k}}{\partial k}\right|_{k_{\mathrm{F}}} \cdot\left(k-k_{\mathrm{F}}\right)
$$

and the group velocity is $v_{\mathrm{g}} \equiv\left|\frac{\partial E}{\partial k}\right|_{k_{\mathrm{F}}} \equiv \hbar k_{\mathrm{F}} / m^{*}$, so that $\rho(\mu) \sim 1 / v_{\mathrm{g}} \sim m^{*}$. We now implement these principles to the antiferromagnetic phases. The hybridized bands, together with the density of states in the bands are schematically represented for the paramagnetic state in Fig. 2.
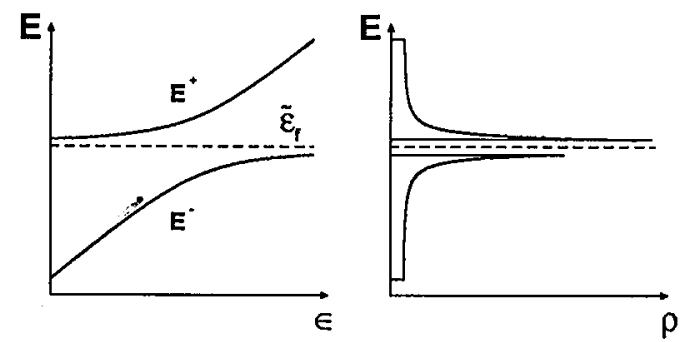

Fig. 2. The quasiparticle bands $E_{k}^{(s)}$, with $s= \pm 1$ (left part). $\tilde{\varepsilon}_{f}$ represents the renormalized $f$-level position. The right part of the figure represents the density of states. The (indirect) gap appears only if $V_{k}$ has no nodes (here $V_{k}=V$ is of intraatomic nature).

\subsection{Case B: antiferromagnelic heavy-fermion phases}

In order to describe the antiferromagnetic phases, we subdivide e.g. a simple cubic lattice into two interpenetrating lattices having magnetic moments oriented 
in opposite directions. This means that the lattice periodicity is broken and the period doubles in such simple antiferromagnetic state. This also means that the first Brillouin zone is reduced and instead of two hybridized bands depicted in Fig. 2, we have in the present situation four subbands, as each of the original bands splits into two subbands of the Slater type. The situation is schematically depicted in Fig. 3 (there are two antiferromagnetic phases, see below). The role of the molecular field is taken over by the staggered molecular field $\beta_{3}$, which is of opposite sign on the two sublattices. In effect, we have the staggered (sublattice) magnetic moment decreasing with increasing hybridization, as shown in Fig. 4.Both the $f$ - and
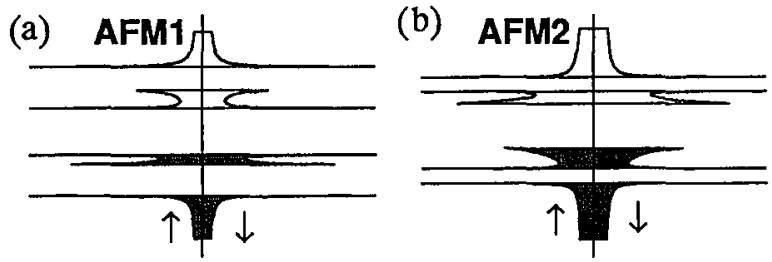

Fig. 3. The density of quasiparticle states in the antiferromagnetic states AFM1 (a) and AFM2 (b). Note that the middle two bands have the shapes reversed one with respect to each other (for details, see main text). Each of the Slater subbands can accommodate up to $N$ particles.

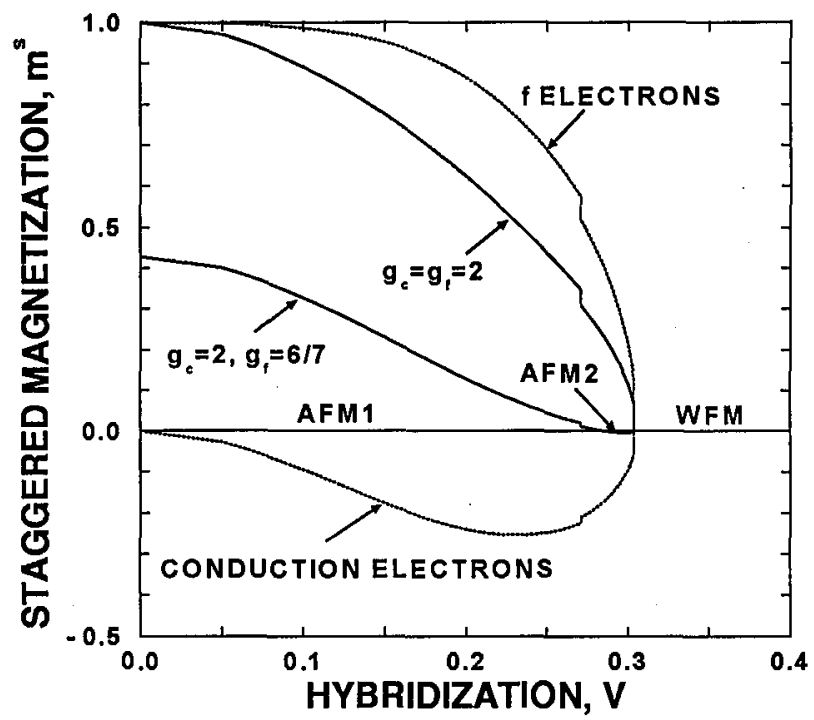

Fig. 4. Staggered (sublattice) magnetization $m^{s}=\frac{1}{2} g_{f} m_{f}^{s}+\frac{1}{2} g_{c} m_{c}^{s}$ in AFM1 and AFM2 phases, as a function of hybridization for $U / W=1.5, \varepsilon_{f} / W=-0.75$, and $n_{\mathrm{e}}=1.99$. The dotted lines represent respectively the $f$ - and $c$-electron contributions. The antiferromagnetism disappears at $V_{\text {AFM2-WFM. }} V$ is in units of $W$. 
$c$-electrons have nonzero staggered moment and, as we see, oriented antiparallel with respect to each other. What is more important, the system effectively demagnetizes for a sufficiently large hybridization. The effect is particularly pronounced for $n=2-\delta$, with $\delta \ll 1$. Note also that antiferromagnetic (AF) phase transforms into weak ferromagnetic phase (WFM), with the polarization $m=2-\delta$. Furthermore, the $f$-electrons autocompensate their moment by acquiring the Fermi-liquid (itinerant) character.

In order to single out the part of the $f$-moment compensated by $f$-electrons themselves, we plotted in Fig. 5 the compensation ratio $r \equiv\left|m_{f}^{\mathrm{s}} / m_{c}^{\mathrm{s}}\right|$ and show that the carrier can reduce the $f$-moment by a half at most. Also, the moment evolves from the atomic value $m_{f}^{\mathrm{s}}=n_{f}=1$, and $m_{\mathrm{c}}^{\mathrm{s}}=0$ at very small hybridization, to zero at $|V| / W \approx 0.3-0.4$. The small discontinuous jump of magnetization to smaller values is associated with the change in the electronic structure in the same way. In that process the bottom and the top of the highest partially (or totally) filled subband reverse (cf. Fig. 3). This change can be seen explicitly in Fig. 6, where the hybridization dependence of the density of states enhancement $\rho / \rho_{0}$ at the Fermi level was plotted for two values of $\delta=2-n=0.01$ and 0.05 (i.e. close to the case with two electrons per atom). For a comparison, the enhancement $\rho / \rho_{0}$ for the paramagnetic phase is shown as the dashed line. In the paramagnetic case the density of states grows when $V \rightarrow 0$, since the $f$-level gradually acquires the full strength. The situation is not that simple for the antiferromagnetic solution, since the corresponding curve is nonmonotonic and the maximum is reached at the point $|V| \approx 0.3 \mathrm{eV}$, i.e. when the magnetic moment is very small. Additionally, the occupancy of the $f$-level is then very close to unity (in the symmetric

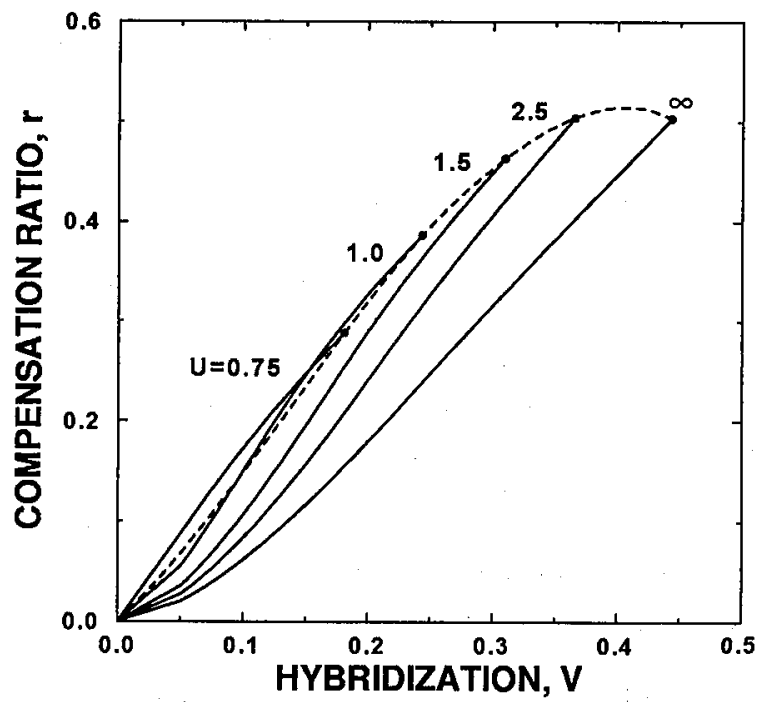

Fig. 5. The compensation ratio $r=\left|m_{c}^{\mathrm{s}} / m_{f}^{\mathrm{s}}\right|$ as a function of hybridization, for different values of $U / W$, and for $\varepsilon_{f} / W=-0.75$. For $U \rightarrow \infty$, the value of $r$ approaches $1 / 2$. $V$ is in units of $W$. 


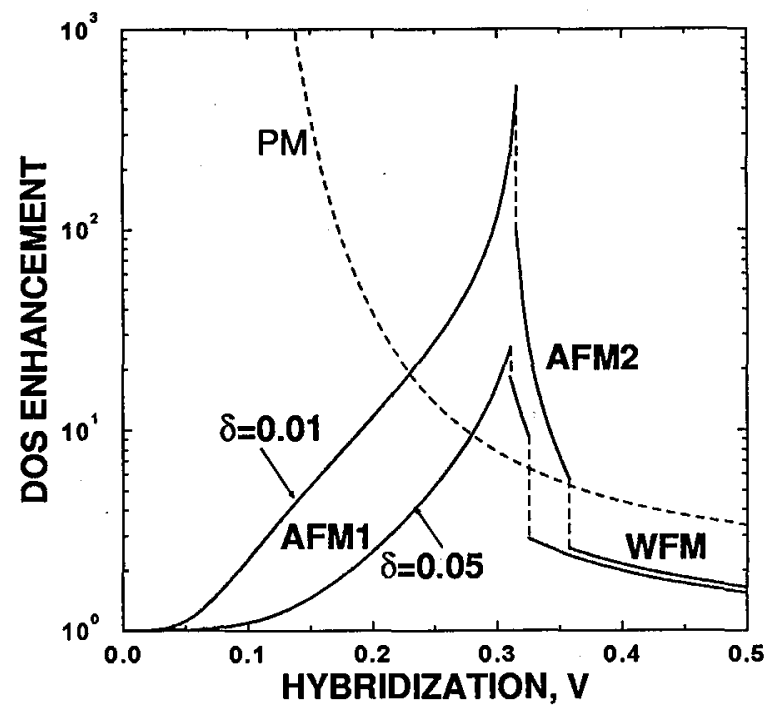

Fig. 6. The density-of-states enhancement $\rho / \rho_{0}$ at the Fermi level for $U / W=2.5$, $\varepsilon_{f} / W=-0.75$, and the number of electrons $n=2-\delta=1.99$ and 1.95 . The dashed line representing the paramagnetic case (PM) is drawn for comparison. The transition from AFM1 to AFM2, and from AFM2 to a. WFM phase are of first order. $V$ means $|V| / W$.

case $2 \varepsilon_{f}+U=0, n_{f}$ is exactly unity). All these feature reproduce very nicely the principal properties of heavy fermions in the antiferromagnetic ordered state. Obviously, within such a simple model, it is impossible to reproduce the anisotropic properties of those systems, as one has to include the crystal-field structure and the $k$ dependence of the hybridization ( $V \rightarrow V_{k}$ with possibility of having it vanishing in certain $k$ directions).

\section{Kondo semiconducting state}

The above feature concerning the moment compensation of the $f$-states applies to the same extent to the Kondo insulating limit, i.e. when $n=2$, therefore in the paramagnetic state the lower hybridized band is filled and the upper is empty. The value of the gap as a function of hybridization has been displayed in Fig. 7 (upper panel) together with the $f$-level filling $n_{f}$ (lower panel), for both antiferromagnetic (solid lines) and paramagnetic (dashed lines) states. The gap can be of the order of $\approx 1 / 4$ of the initial band width $W$, but for $|V|<0.25$ is rather small.

Most interesting features of the Kondo semiconducting state appear at nonzero temperature $(T>0)$, when the gap gradually reduces to zero at temperature $T_{0}$ in the paramagnetic state. In Fig. 8 we plotted the temperature $\left(T / T_{0}\right)$ dependence of the reduced gap $\Delta(T) / \Delta(0)$, for three values of hybridization $|V| / W=0.16,0.18$, and 0.20 and compared it with the data [9] for $\mathrm{FeSi}$, for which $\Delta(0) \approx 50 \mathrm{meV}$, and $T_{0}=200 \mathrm{~K}$. The theoretical curves are not universal, but they do fit quite with the trend of the data. Particularly important is the linear $(\Delta(T) \sim T)$ dependence of the gap when approaching $T_{0}$. 


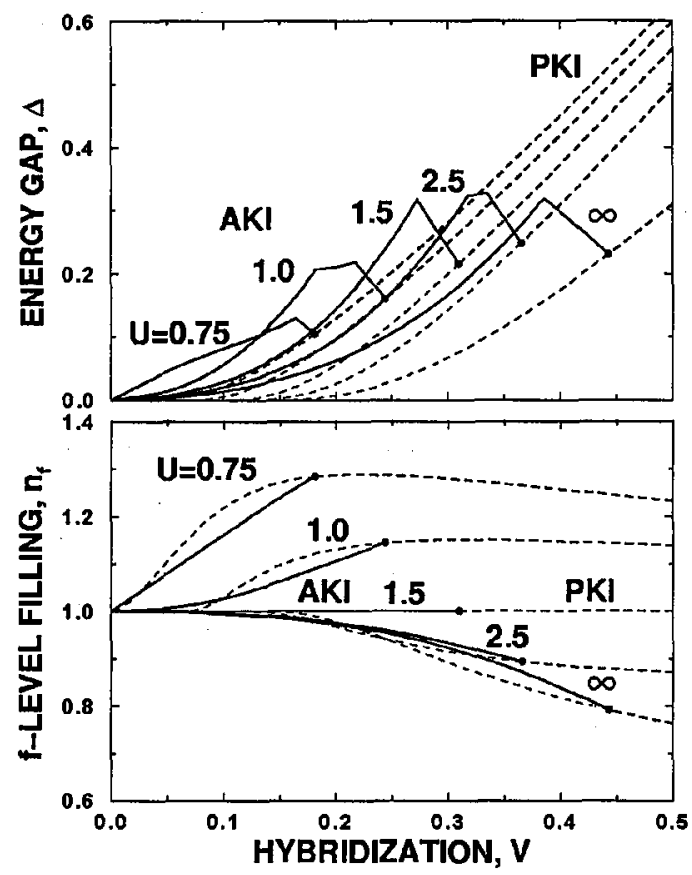

Fig. 7. The hybridization gap in the quasiparticle spectrum (top) and the $f$-level occupancy (bottom), both as a function of hybridization, for $\varepsilon_{f} / W=-0.75, n=2$, and various $U$ values. Solid lines - antiferromagnetic Kondo insulating phase (AKI), dashed lines - paramagnetic phase (PKI). $\Delta$ is in units of $W$.

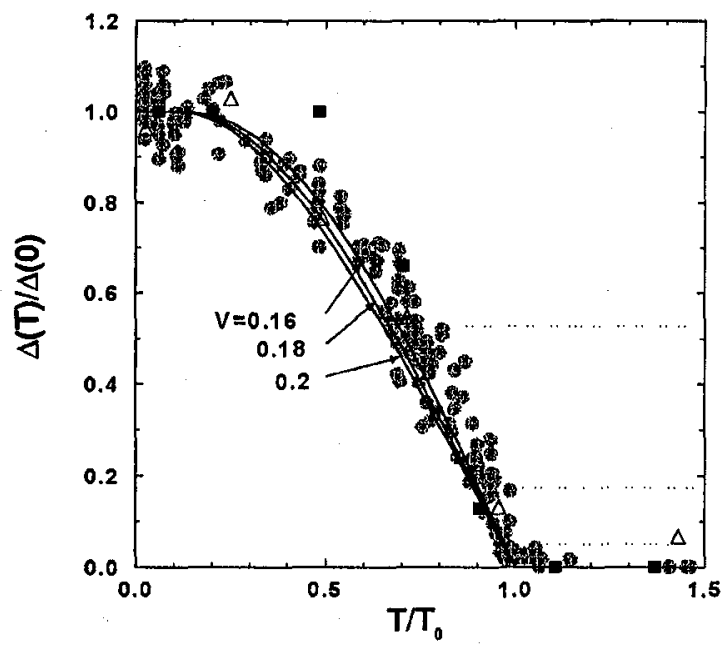

Fig. 8. The temperature dependence of the semiconducting gap: solid line - theory, circles - tunneling spectroscopy (Ref. [9]), squares - Raman spectroscopy (Ref. [10]), triangles - infrared spectroscopy (Ref. [11]). 
The physical picture emerging in this approach as $T \geq T_{0}$ is as follows. The $f$-level occupancy $n_{f} \rightarrow 1$ and thus $\tilde{V} \rightarrow 0$. On the other hand, $\varepsilon_{f}+$ $\beta_{0}-\mu \rightarrow 0$, and hence the $f$-level is placed exactly at the Fermi level, but the localized $f$-states do not hybridize with those of carriers. The separation with increasing temperature into the two separate subsystems is due to the fact that the thermal fluctuations disrupt the quantum coherence of the quasiparticle states. This means that the Fermi-liquid state with heavy quasiparticles is unstable if thermodynamic fluctuactions are taken into account. One should mention, however, that within the saddle-point approximation employed in the discussion here one obtains an incorrect value of the entropy of $f$-electrons for $T>T_{0}$ [5].

\section{Conclusions}

The Kondo semiconductors (insulators at $T=0$ ) represent a new class of semiconductors, since their gap gradually reduces to zero with increasing temperature $T \rightarrow T_{0}$. The temperature $T_{0}$ is not related to any magnetic transition temperature or any other long-range ordering. It is related to the temperature at which the disruption of quantum coherence of the itinerant $f$-states becomes complete. A similar phenomenon takes place in the almost localized metallic Mott-Hubbard systems, where the Mott transition takes place to the semiconducting phase at

(a)

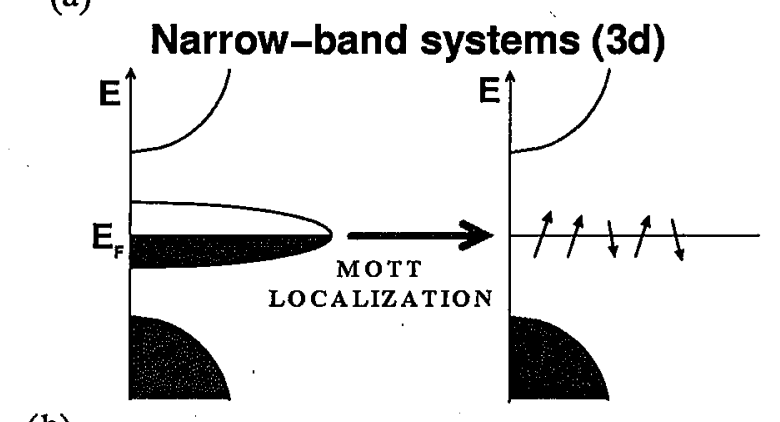

(b)

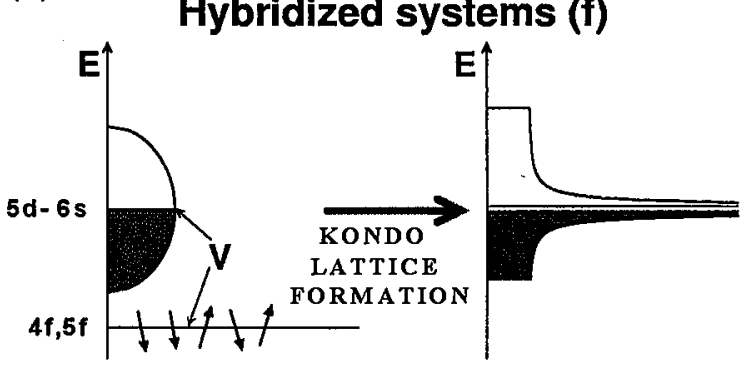

Fig. 9. Schematic illustration of a fundamental difference between the Mott localization (to a magnetic semiconductor state) (a) and the Kondo-insulator (correlated band semiconductor) formation (b). 
$T>0$ [12], which is of first order. However, there is a principal difference between the Kondo-lattice insulators and Mott-Hubbard systems, as schematically depicted in Fig. 9. Namely, the former are essentially the band insulators with the decreasing gap growing with $T$, whereas the latter have a well defined magnetic moment in the insulating phase (i.e. are magnetic semiconductors). Nonetheless, we still need a better quantitative understanding of the changeover from the Fermi liquid or the Kondo insulator to the state at $T>T_{0}$ (or $T>T_{\mathrm{K}}$ in the heavy-fermion metallic case), in which the $f$-electrons are represented as localized spins, even though they are itinerant in the ground-state configuration.

\section{Acknowledgment}

The authors acknowledge the financial support through grant No. 2P03B 12912 of the Committee for Scientific Research. R.D. was also supported by the special grant for graduate students No. 2P03B 10815.

\section{References}

[1] F. Steglich, J. Aarts, C.D. Bredl, W. Lieke, D. Meschede, W"Franz, H. Schäfer, Phys. Rev. Lett. 43, 1892 (1979).

[2] T. Takabatake, F. Teshima, H. Fuji, S. Nishigori, T. Suzuki, T. Fujita, Y. Yamaguchi, J. Sakurai, Phys. Rev. B 41, 9607 (1990); G. Aeppli, Z. Fisk, Comments Condens. Matter Phys. 16, 155 (1992).

[3] B. Andraka, G.R. Stewart, Phys. Rev. B 47, 3208 (1993); H. v. Löhneysen, T. Pietrus, G. Portisch, H.G. Schlager, A. Schröder, M. Sieck, T. Trappmann, Phys. Rev. Lett. 72, 3262 (1994).

[4] R. Doradziński, J. Spałek, Phys. Rev. B 56, R14 239 (1997); ibid. 58, 3293 (1998); Physica B 259-261, 170 (1999); J. Spałek, R. Doradziński, in: Magnetism and Electronic Correlations in Local-Moment Systems: Rare-Earth Elements and Compounds, Eds. M. Donathi, P.A. Dowben, W. Nolting, World Scientific, Singapore 1998 , p. 387.

[5] R. Doradziński, Ph.D. thesis, Warsaw University, 1999 (unpublished).

[6] J. Flouquet, P. Kambe, private communication.

[7] J.R. Schrieffer, P.A. Wolff, Phys. Rev. 149, 491 (1966).

[8] J. Kondo, Prog. Theor. Phys. (Kyoto) 32, 37 (1964).

[9] M. Fäth, J. Aarts, A.A. Menovsky, G.J. Nieuwenhuys, J.A. Mydosh, Phys. Rev. $B$ 58, 15483 (1998).

[10] P. Nyhus, S.L. Cooper, Z. Fisk, Phys. Rev. B 51, 15626 (1995).

[11] A. Damascelli, K. Schulte, D. van der Marel, A.A. Menovsky, Phys. Rev. B 55, R4863 (1997).

,[12] J. Spałek, A. Datta, J.M. Honig, Phys. Rev. Lett. 59, 728 (1987); for a didactical review see: J. Spałek, J. Solid State Chem. 88, 70 (1990); see also: A. Georges, G. Kotliar, W. Krauth, M.J. Rozenberg, Rev. Mod. Phys. 68, 13 (1996). 